\title{
A common error term regulates acquisition but not extinction of causal judgments in people
}

\author{
Oren Griffiths • R. Frederick Westbrook
}

Published online: 27 December 2011

(C) Psychonomic Society, Inc. 2011

\begin{abstract}
In three experiments, we used the allergist task to examine the role of error correction mechanisms in the acquisition and extinction of causal judgments in people. Consistent with existing human and animal studies, acquisition of causal judgments was influenced by the discrepancy between the allergenic outcome and that predicted by all of the cues present on a trial (the "common error" term). However, in the present experiments, we failed to detect any evidence for the use of a common error term in extinction learning: Judgments of the allergenic properties of a cue were unaffected by the predictive value of the other cues present on a trial. This asymmetry in the use of a common error term in acquisition and extinction learning is inconsistent with previous animal studies and also with most models of associative learning. However, approaches that allow learning to be specific to a particular arrangement of elemental cues (context specific and state based) offer some explanation of the observed asymmetry.
\end{abstract}

Keywords Human causal learning - Extinction · Associative learning

Contemporary theories of associative learning invoke error correction mechanisms to explain a range of Pavlovian acquisition phenomena in animal subjects. These theories originate in the blocking, contingency, and signal validity effects reported in the classic experiments by Kamin (1969), Rescorla (1969), and Wagner (1969), respectively. Each of these experiments demonstrated that learning about a conditioned stimulus (CS X) depends not only on its relation to

O. Griffiths $(\bowtie) \cdot$ R. F. Westbrook

School of Psychology, University of New South Wales,

Sydney 2052, Australia

e-mail: oren.griffiths@unsw.edu.au a motivationally significant outcome [an unconditioned stimulus (US)] but also on the relation between other concomitantly present cues (CSA) and the US. More specifically, each demonstrated that a relation between $\mathrm{X}$ and the US, which was normally effective in producing learning, was rendered ineffective by the presence of a better predictor (A) of the US. Learning about X was reduced when pairings of an AX compound and the US were preceded by pairings of A and the US (Kamin, 1969), when X-US pairings were interspersed with US-alone presentations in the context (A) where the pairings occurred (Rescorla, 1969), and when X was paired with the US in the presence of A but not $\mathrm{B}$ relative to when $X$ was equally often paired or not paired with the US in the presence of A and B (Wagner, 1969). These effects led to the development of theories (e.g., Pearce \& Hall, 1980; Rescorla \& Wagner, 1972; Wagner, 1981) whose central proposals are that associative formation is regulated by the discrepancy between the actual and predicted outcome of a trial and that all of the cues present are used to calculate the discrepancy whose size determines associative change. These theories have not only explained the blocking, contingency, and signal validity effects as well as other existing Pavlovian acquisition phenomena (e.g., overshadowing and conditioned inhibition), but have also successfully predicted new ones (e.g., over expectation and super conditioning).

Dickinson, Shanks, and Evenden (1984) noted the similarity between the procedures used to study associative learning in animals and judgments of causality by people. They proposed, therefore, that the theories developed to explain how animals learn about relations between events in Pavlovian conditioning procedures also characterize how people acquire this knowledge in causal learning tasks. There is evidence for this proposal. Several investigators (e.g., Dickinson, 2001; Le Pelley \& McLaren, 2003; Le 
Pelley, Oakeshott, Wills, \& McLaren, 2005; Van Hamme \& Wasserman, 1994) have reported that learning about causal relations in people exhibits many of the characteristics observed in conditioning studies with animals. Such characteristics include blocking, conditioned inhibition, and super conditioning: The relation between a cue $(\mathrm{X})$ and an outcome that normally produces judgments of causality is ineffective when $\mathrm{X}$ is presented in compound with a cue (A) that already predicts the outcome (Aitken, Larkin, \& Dickinson, 2001); a cue (B) that signals the absence of an outcome otherwise predicted by A becomes a conditioned inhibitor or a preventative cause of the outcome (Chapman \& Robbins, 1990); and judgments about the causal effectiveness of a novel cue $(\mathrm{X})$ are increased when an outcome follows a compound composed of $\mathrm{X}$ and a cue (B) that had been trained as a conditioned inhibitor or preventative cause (Aitken et al., 2000).

Contemporary models of associative learning make an additional claim, which is that a common error term also regulates associative change in extinction. Here, the omission of the predicted outcome constitutes the error, which drives new associative formation - either an inhibitory CSUS association (Rescorla, 1979) or an excitatory association between the CS and some sort of "no outcome" representation (Bouton, 1993; Konorski, 1968). Evidence for a common error term in extinction learning with animal subjects was provided by Wagner (1969), who found that a moderately trained cue underwent a greater depression of responding when extinguished in compound with a well-trained than with a weakly trained cue. Similarly, Rescorla (2000) reported that a cue extinguished in compound with another trained cue could, depending on parameters, undergo a greater depression in conditioned responding than a cue extinguished in isolation. Rescorla (2003) also found that response loss to a cue extinguished in compound with a familiar but neutral stimulus was greater than that to a cue extinguished in compound with a conditioned inhibitor. Finally, Leung and Westbrook (2008) reported greater loss of responding to a cue that was extinguished in compound with an extinguished cue undergoing spontaneous recovery (error was large) than to a second cue extinguished in compound with an extinguished cue not undergoing recovery (error was small).

As was noted previously, there is evidence that a common error term regulates the acquisition of causal judgments in people, but - as far as we are aware - the role of a common error term in the extinction of these judgments has not been studied. Therefore, in three experiments we examined whether people, like animals, use a common error term to regulate not only acquisition but also extinction of cue-outcome associations in a causal learning task. In each experiment, we used a task in which participants were required to assume the role of an allergist who is trying to determine which foods produce an allergy in his or her patients (e.g., Larkin, Aitken \& Dickinson, 1998).

\section{Experiment 1}

In Experiment 1, we used a two-part allergist task in which participants were given two patients to learn about (Mr. X and Mr. Y), one at a time. The schedule of foods and allergies experienced by $\mathrm{Mr}$. X and Mr. Y examined the role of a common term in extinction and acquisition of a learned allergy, respectively. The foods eaten (the cues) were specific to each patient so as to minimize generalization, and the order in which participants learned about the two patients was counterbalanced. The design is shown in Table 1.

In the case of Mr. X, particpants learned in Phase 1 that seven foods $(A+$ to $\mathrm{G}+)$ were dangerous, whereas four foods ( $\mathrm{H}-$ to K-) were safe. In Phase 2, A was presented in the absence of the allergy (A-), whereas $\mathrm{F}+$ and $\mathrm{G}+$ remained dangerous. In Phase 3, the already extinguished A was presented in compound with the dangerous $\mathrm{B}$ and the compound was not followed by the allergy (AB-), whereas a second compound, composed of two other dangerous foods, was also presented and was not followed by the allergy (CD-). Finally, participants were asked to judge which of two meals was more likely to produce an allergy (BF vs. DG) and to rate the effectiveness of each meal (A to $K$ ) in producing an allergy. The rationale of this design is that the presence of the already extinguished $A$ in compound with $B$ across extinction of the $\mathrm{AB}$ compound in Phase 3 will allow participants to predict the outcome (no allergy) more quickly than they will predict that the compound composed of two dangerous foods $(\mathrm{CD})$ is no longer followed by the allergy. That is, error will be less across the AB- trials than the CDtrials, with the consequence that participants will learn less about the relation between $\mathrm{B}$ and the new outcome (no allergy) than about D (or C) and the new outcome. Hence, they should select a meal containing a dangerous food $(\mathrm{F})$ and the less-well-extinguished $\mathrm{B}$ as more dangerous than a meal containing a dangerous food $(\mathrm{G})$ and the better extinguished D. Similarly, they should rate B as more effective in producing an allergy than $\mathrm{D}$ ( or C).

In the case of Mr. Y, participants learned in Phase 1 that seven foods were safe to consume (L- to R-), whereas four were dangerous $(\mathrm{S}+$ to $\mathrm{V}+$ ). In Phase 2 , one of the safe foods was followed by allergy $(\mathrm{L}+)$, whereas two remained safe (Q-, R-). In Phase 3, one of the now dangerous foods $(\mathrm{L}+)$ was combined with a previously safe food $(\mathrm{M}-)$ and the compound was followed by the allergy (LM+), whereas a compound composed of two previously safe foods ( $\mathrm{N}$ - and $\mathrm{O}-$-) was also followed by the allergy $(\mathrm{NO}+)$. Finally, participants were asked to judge which of two meals was more likely to 
Table 1 Design of Experiment 1

\begin{tabular}{|c|c|c|c|c|c|c|c|c|c|}
\hline \multicolumn{5}{|l|}{ Mr. X } & \multicolumn{5}{|l|}{ Mr. Y } \\
\hline Phase 1 & Phase 2 & Phase 3 & Test 1 & Test 2 & Phase 1 & Phase 2 & Phase 3 & Test 1 & Test 2 \\
\hline $\mathrm{A}+$ & A- & AB- & BF Vs. DG & $\begin{array}{l}\text { Causal } \\
\quad \text { Rating (A-K) }\end{array}$ & L- & $\mathrm{L}+$ & $\mathrm{LM}+$ & MQ vs. OR & $\begin{array}{l}\text { Causal } \\
\text { Rating (L-V) }\end{array}$ \\
\hline $\mathrm{B}+$ & & & & & M- & & & & \\
\hline $\mathrm{C}+$ & & CD- & & & $\mathrm{N}-$ & & $\mathrm{NO}+$ & & \\
\hline $\mathrm{D}+$ & & & & & $\mathrm{O}-$ & & & & \\
\hline $\mathrm{E}+$ & E- & & & & P- & $\mathrm{P}+$ & & & \\
\hline $\mathrm{F}+$ & $\mathrm{F}+$ & & & & Q- & Q- & & & \\
\hline $\mathrm{G}^{+}$ & $\mathrm{G}^{+}$ & & & & $\mathrm{R}-$ & $\mathrm{R}-$ & & & \\
\hline H- & $\mathrm{H}-$ & $\mathrm{HJ}+$ & & & $\mathrm{S}+$ & $\mathrm{S}+$ & SU- & & \\
\hline I- & $\mathrm{I}+$ & $\mathrm{IK}+$ & & & $\mathrm{T}+$ & $\mathrm{T}-$ & TV- & & \\
\hline $\mathrm{J}-$ & & & & & U+ & & & & \\
\hline K- & & & & & $\mathrm{V}+$ & & & & \\
\hline
\end{tabular}

Letters A-V denote cues, and “+” and “-” indicate reinforced and nonreinforced trials, respectively. The distinction between the acquisition/ extinction and filler components is presented for ease of explanation only; participants witnessed all trial types within each phase presented in a randomly intermixed fashion

produce an allergy (MQ versus $\mathrm{OR}$ ) and then to rate the effectiveness of each food ( $\mathrm{L}$ to $\mathrm{V}$ ) in producing an allergy. The rationale is that the presence of the already trained food $(\mathrm{L}+)$ in the compound with $\mathrm{M}(\mathrm{LM}+)$ will allow participants to predict the allergenic outcome more quickly than when a compound composed of two previously safe foods is followed by the allergy $(\mathrm{NO}+)$. Because error will be less across the $\mathrm{LM}+$ trials than across the $\mathrm{NO}+$ trials, participants will learn less about the relation between $\mathrm{M}$ and the allergy than about $\mathrm{O}$ (or N) and the allergy (that is, L will block M). Hence, they should select a meal containing a dangerous food $(\mathrm{Q})$ and the less-well-conditioned $\mathrm{M}$ as less dangerous than a meal containing a dangerous food (R) and the better conditioned $\mathrm{O}$. Similarly, they should rate $M$ as less effective in producing an allergy than $\mathrm{O}$ (or $\mathrm{N}$ ).

The other foods in the design constituted a further test of the role played by error-correction mechanisms in acquisition ( $\mathrm{H}$ to $\mathrm{K}$ for Mr. $\mathrm{X}$ ) and extinction (S to $\mathrm{V}$ for $\mathrm{Mr}$. Y) of causal judgements. If a common error term influences the amount learned in acquisition, then participants will rate $\mathrm{K}$ as less causal than $\mathrm{J}$, since $\mathrm{K}$ was combined into an allergenic compound with the already trained I in Phase 3, whereas neither $\mathrm{J}$ nor $\mathrm{H}$ was allergenic prior to being combined into a compound that signalled the allergy. Thus, the dangerous cue I should block learning that $\mathrm{K}$ is dangerous since cue I already predicts the allergy across the IK+ trials. If a common error term influences the amount learned in extinction, then participants will rate $\mathrm{V}$ as more causal than $\mathrm{U}$, since the dangerous $\mathrm{V}$ was extinguished in compound with the already extinguished $\mathrm{T}$ in Phase 3 , whereas $\mathrm{U}$ was extinguished in compound with the dangerous $\mathrm{S}$. Thus, the already extinguished $\mathrm{T}$ should block learning that $\mathrm{V}$ is safe, since $\mathrm{T}$ already predicts the nonoccurrence of the allergy across the TV trials.

Any differential influence of base rate on acquisition and extinction learning was controlled by assigning acquisition and extinction training to separate patients, and then by using the remaining (filler) cues to test the opposite type of learning in that patient. For example, in the patient that tested extinction learning (Mr. X), the filler cues tested the role of a common error term on acquisition learning (and vice versa for Mr. Y).

\section{Method}

Participants A total of 233 undergraduate students (mean age 20 years) participated in partial fulfilment of a laboratory requirement in a psychology course.

Procedure Participants were instructed that they were to assume the role of an allergist, and that they would be required to learn which foods made two new patients (Mr. X and Mr. Y) feel ill, and which were safe for him to consume. They were told that they would deal with only one patient at a time. During the delivery of the instructions, they were given two practice trials (with a third patient, Mr. Z, who ate different foods than Mr. X and Mr. Y). They then proceeded to Phase 1. On each trial, participants were shown an individual food (e.g., the word carrots) and were asked to predict whether Mr. X would feel ill. Food (e.g., carrot) was randomly assigned to cue types (e.g., A) for each participant (a list of the foods used in the present studies is provided in Appendix A1). 
Two response keys were available, labeled Sick and Healthy. The selection of a response key resulted in a picture of the patient feeling nauseated $(+)$ or of the patient feeling healthy $(-)$. If an incorrect prediction was made, the word "incorrect" was shown in red, a beep tone sounded, and the correct answer was shown for 2 s. If a correct prediction was made, the word "correct" was shown in green, and no tone or time penalty occurred. The order of the trials in each phase was randomized with the constraint that all trial types (e.g., A+, L-, etc.) were shown once before any trial type was shown a second time. There were eight instances of each trial type in each phase. The transition between phases was not signaled.

Upon completing training with $\mathrm{Mr}$. X, participants were given a test phase in which they were asked questions about their experience with Mr. X. This started with a forced choice test, based on Rescorla's (2001) compound test procedure (see Table 1). They were shown a picture of Mr. X experiencing an allergic reaction in the middle of the screen, and two meals (each consisting of two foods, BF and DG) were listed on the bottom right and bottom left of the screen, respectively. Participants were asked to select which of the two meals was more likely to have produced Mr. X's allergic reaction. The assignment of foods to the left and right buttons and the order in which the two foods in each meal were shown (e.g., $G$ and $D$, or D and $G$ ) were randomly determined for each participant. This was followed by a causal rating test of each of the 11 foods presented in a random order. On each trial in this test, an image of Mr. X feeling nauseated and a single training cue were shown to participants. For each cue, they were asked to rate on a scale from 0 (very unlikely) to 100 (very likely) how likely it was that Mr. X would have an allergic reaction after eating that food.

Upon completion of the Mr. X test phase, participants began training with their second patient, Mr. Y. After the Mr. $\mathrm{Y}$ training, participants were asked a series of questions about their experience with that patient, in a manner identical to the Mr. X test phase. Mr. X was the first patient for half of the participants, and Mr. Y for the remainder.

\section{Results}

Training data Prediction data for the target cues across Phases 1-3 are shown in the Fig. 1a (Mr. X) and Fig. 1b (Mr. Y). In Phase 1, participants quickly discriminated between those foods that caused an allergy (A - G for Mr. X and $\mathrm{L}-\mathrm{R}$ for $\mathrm{Mr}$. $\mathrm{Y}$ ) and those that were safe for each patient to consume ( $\mathrm{H}-\mathrm{K}$ for $\mathrm{Mr}$. X and $\mathrm{S}-\mathrm{V}$ for $\mathrm{Mr}$. $\mathrm{Y})$. In Phase 2, the changed contingencies produced a transient reduction in the accuracy with which participants predicted that foods $\mathrm{F}$ and $\mathrm{G}$ were followed by the allergy for Mr. X,
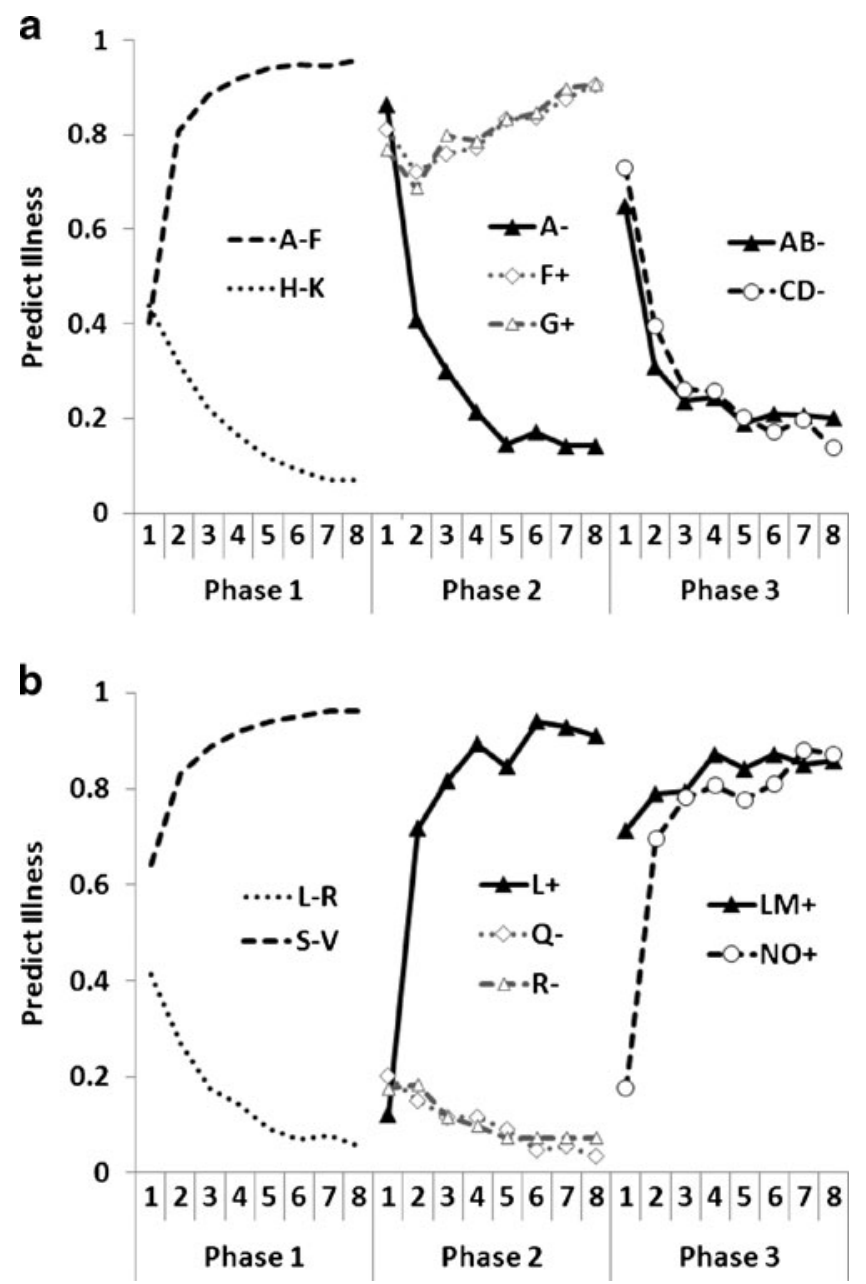

Fig. 1 Mean outcome predictions in training phases 1-3 in Experiment 1. a outcome predictions for the extinction patient (Mr. X). b outcome predictions for the acquisition patient (Mr. Y)

but these predictions recovered to the levels that they had obtained at the end of Phase 1. Participants quickly reduced their predictions about the allergenic consequences of food $\mathrm{A}$, and their predictions that A was not followed by an allergy remained low across the final few trials. Participants quickly learned that food $\mathrm{L}$ was dangerous while continuing to predict that foods $\mathrm{Q}$ and $\mathrm{R}$ remained safe for Mr. Y. In Phase 3, participants did not appear to use the information acquired about $\mathrm{A}$ across its extinction to predict the outcome (no allergy) when it was combined with the allergenic B in the case of Mr. X; they learned that a compound composed of two allergenic foods was not followed by the allergy (CD-) at a similar rate as they learned that a compound composed of the already extinguished $\mathrm{A}$ and the allergenic B (AB-) was not followed by the allergy. In contrast, participants continued to predict with high accuracy that a compound composed of the dangerous $\mathrm{L}$ and the previously safe $\mathrm{M}$ was followed by the allergy $(\mathrm{LM}+)$, but quickly learned that a compound composed of two previously safe foods was now followed by the allergy $(\mathrm{NO}+)$. To examine 
whether there were any differences in the rate at which participants learned about the compounds in Phase 3, an additional analysis was performed upon the training data. Specifically, within the extinction component in the case of Mr. X, the number of participants who predicted the correct outcome for each compound ( $\mathrm{AB}$ or $\mathrm{CD}$ ) was summed separately for each trial (1-8). These frequency values were entered into a chi-square procedure that compared prediction accuracy for compound $\mathrm{AB}$ against $\mathrm{CD}$, across the eight trials of Phase 3. No significant differences between the compounds were found in the pattern of prediction accuracies, $\chi(7)=4.54, p>.05$. Thus, we did not find any evidence that extinction occurred at different rates in these compounds. The same analysis was performed upon the Phase 3 data from the acquisition component in the case of Mr. Y. A significant difference was seen in the rates at which the correct outcome was predicted on $\mathrm{LM}+$ and $\mathrm{NO}+$ trials, $\chi(7)=75.52$, $p<.001$. Participants were very accurate in their predictions on the initial $\mathrm{LM}+$ trials, but needed approximately three trials to reach this level of accuracy on $\mathrm{NO}+$ trials.

Forced choice data A binomial test on the forced choice data revealed that participants were indifferent when asked to choose which of two meals (BF and $\mathrm{DG}$ ) was more dangerous to Mr. X (116 out of 233 selected $\mathrm{BF}, \mathrm{z}=-0.07$, $p>.05$ ). Both meals contained an allergenic ( $\mathrm{F}$ and $\mathrm{G})$ and an extinguished (B and D) food, differing in that $\mathrm{B}$ had been extinguished in compound with the already extinguished A, whereas D had been extinguished in compound with another allergenic food C. In contrast, participants selected OR as more dangerous than MQ to Mr. Y (148 out of $233, \mathrm{z}=4.13$, $p<.001)$. Q and $\mathrm{R}$ had each been reinforced and then extinguished, while $\mathrm{M}$ and $\mathrm{O}$ had both been continuously reinforced. However, $\mathrm{M}$ had been trained in compound with the already trained $\mathrm{L}$, whereas $\mathrm{O}$ had been trained in compound with a previously safe $\mathrm{N}$.

Causal ratings data The causal rating data for each cue is shown in Fig. 2. These data were analyzed using a set of orthogonal contrasts. The ratings of the cues $(\mathrm{L}-\mathrm{R})$ for the acquisition component (Mr. Y) are described first. The cue paired with the allergy in Phases 2 and 3 (L), was rated as significantly more causal than the average of the cues that had each been paired with the allergy in a single phase $(\mathrm{M}, \mathrm{N}$, and $\mathrm{O}), F(1,231)=160.83, p<.001, \eta_{\mathrm{P}}{ }^{2}=0.410$. Importantly, cue $\mathrm{M}$, trained in compound with the pretrained L in Phase 3, was rated as significantly less causal than $\mathrm{N}$ and $\mathrm{O}$, which were trained in a compound in Phase 3, $F(1,231)=15.44, p<.001, \eta_{\mathrm{P}}{ }^{2}=0.063$. A 95\% confidence interval derived from this contrast showed that cue $\mathrm{M}$ was rated lower than cues $\mathrm{N}$ and $\mathrm{O}$ by, on average, between 4.51 and 13.58 points on the rating scale. Finally, $\mathrm{Q}$ and $\mathrm{R}$ did not significantly differ, $F(1,231)=1.99, p=.16$, suggesting that the preference for OR over MQ was due to the difference between $\mathrm{M}$ and $\mathrm{O}$.

The same contrasts were used to analyze the ratings of the cues (A-F) for the extinction component (Mr. X). The cue extinguished in both Phases 2 and 3 (A) was rated as significantly less causal than the average of the cues $(\mathrm{B}, \mathrm{C}$, and $\mathrm{D})$ that had each been extinguished in a single phase, $F(1,231)=15.37, p<.001, \eta_{\mathrm{P}}{ }^{2}=0.062$. Importantly, cue B that was extinguished in a low prediction error compound (AB) was not rated significantly differently than cues $\mathrm{C}$ and $\mathrm{D}$ that were extinguished in a high prediction error compound (NP), $F<1$. The $95 \%$ confidence interval based upon this contrast revealed that cue B was rated between 2.69 units higher than, and 5.22 units lower than, the average of cues $\mathrm{C}$ and D. Finally, there were no significant differences between the other two cues that were also shown in the forced choice test (cues $\mathrm{F}$ and $\mathrm{G}), F<1$.

The filler cues provided another measure of the role of a common error term in acquisition (Mr. X) and extinction (Mr. Y). Thus, two further orthogonal contrasts were conducted. Cue $\mathrm{J}$ was rated as significantly more causal than cue $\mathrm{K}, F(1,231)=7.26, p=.008, \eta_{\mathrm{P}}{ }^{2}=0.030$, confirming that a common error term regulates acquisition. In contrast, there was no significant difference in ratings between cues $\mathrm{U}$ and $\mathrm{V}, F(1,231)=1.06, p=.30$, confirming the failure to detect evidence that a common error term regulates extinction. Although no difference was seen between cues $\mathrm{U}$ and $\mathrm{V}$, a final contrast revealed that our prediction error manipulation was effective, since the partners of these cues ( $\mathrm{S}$ and $\mathrm{T}$ ) were rated significantly differently at test, $F(1,231)=57.45$, $p<.001, \eta_{\mathrm{P}}^{2}=0.199$. The twice reinforced and singly extinguished cue $\mathrm{S}$ was rated as more causal than the singly reinforced and twice extinguished cue $\mathrm{T}$.

There were no statistically significant differences between the causal ratings in the two task order counterbalancing groups (Mr. X first or Mr. Y first), $F<1$, and this betweengroups contrast did not significantly interact with any of the other contrasts, maximum $F(1,231)=3.10$.

\section{Discussion}

With three measures, we confirmed that acquisition of causal judgements is regulated by a common error term. First, participants chose compound OR as more causal than compound MQ. The only difference between these test compounds was that $\mathrm{M}$ had been trained in compound with a cue (L) that predicted the outcome, whereas $\mathrm{O}$ had been trained in compound with one $(\mathrm{N})$ that did not. Thus, $\mathrm{L}$ reduced or blocked the amount learned about $\mathrm{M}$ relative to the effect of $\mathrm{N}$ on $\mathrm{O}$. Second, participants rated $\mathrm{N}$ and $\mathrm{O}$ as more causal than $\mathrm{M}$, confirming that $\mathrm{L}$ had blocked $\mathrm{M}$. Third, participants rated $\mathrm{J}$ as more causal than $\mathrm{K}$, again confirming that 
Fig. 2 Mean causal ratings at test in Experiment 1. The critical comparisons are represented by dark and striped columns

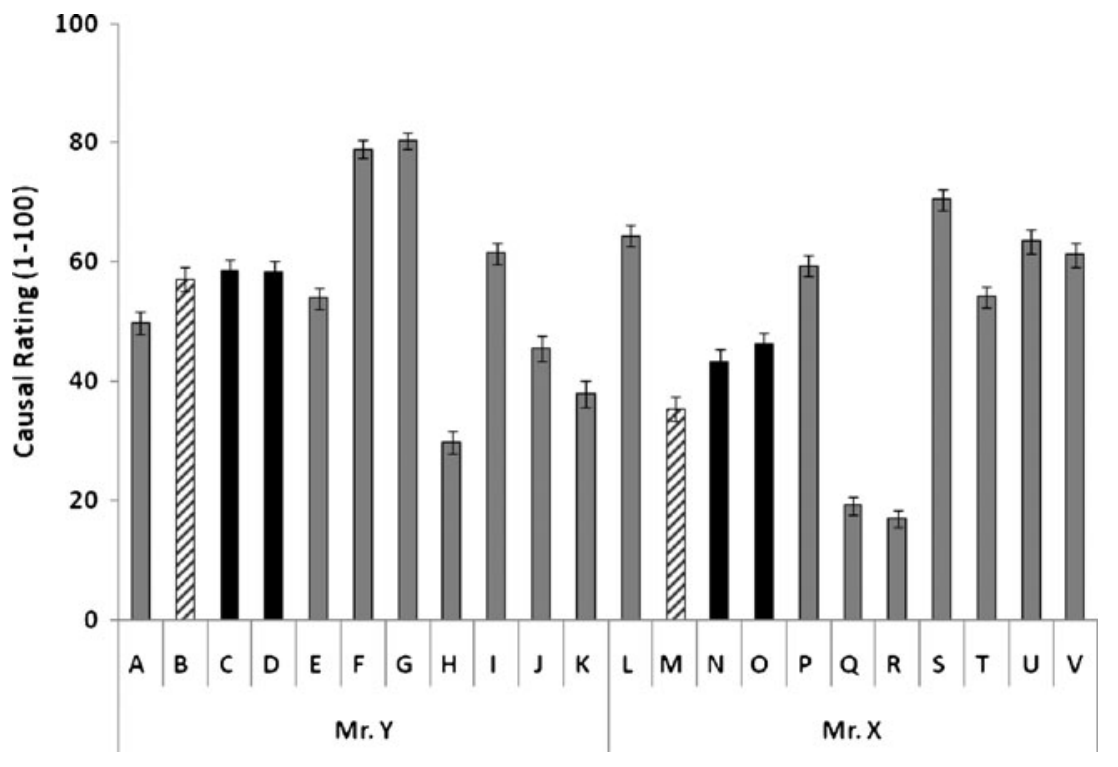

the predictive I had reduced the amount learned about $\mathrm{K}$ relative to the effect of the safe $\mathrm{H}$ on the amount learned about $\mathrm{J}$ across the $\mathrm{HJ}+$ and $\mathrm{IK}+$ trials. These results thus constitute a further demonstration of blocking when assessed by ratings of the causal effectiveness of the individual cues (e.g., Shanks, 1985). They also show such an effect when assessed by a forced choice between two compounds, each of which containing a trained cue and either the blocked cue (M) or its relevant control $(\mathrm{O})$.

However, the failure to find an equivalent blocking effect in human extinction learning is a novel result. Specifically, none of the three test measures provided evidence that extinction is regulated by a common error term. First, participants were indifferent to BF versus DG, in spite of the fact that $\mathrm{B}$ had been extinguished in compound with the already extinguished A, whereas D was extinguished in compound with the nonextinguished C. Second, participants did not rate $\mathrm{B}$ as more causal of the outcome than $\mathrm{C}$ and $\mathrm{D}$. This confirms that the already extinguished cue (A) had failed to block learning about $\mathrm{B}$ across the compound extinction trials, in spite of the fact that A was rated as less causal than B, C, and D. Third, participants did not rate $U$ as less causal than $\mathrm{V}$, although $\mathrm{U}$ had been extinguished in compound with the nonextinguished $\mathrm{S}$, whereas $\mathrm{V}$ had been extinguished in compound with the already extinguished $\mathrm{T}$. This confirms that cue $\mathrm{T}$ had failed to block learning about $\mathrm{V}$ across the compound extinction trials relative to the effect of $\mathrm{S}$ on $\mathrm{U}$ across these trials. Again, this failure was in spite of the fact that the cue T, extinguished in isolation in Phase 2 and in compound with V in Phase 3, was rated as less causal than the cue $\mathrm{S}$, extinguished in compound with $\mathrm{U}$ in Phase 3.

The failure to detect evidence of a role for a common error term in extinction is unlikely to be due to a lack of power. The confidence interval constructed around the contrast comparing cue $\mathrm{B}$ with cues $\mathrm{C}$ and $\mathrm{D}$ was precise enough to be sensitive to a small effect (0.2 $\sigma$ units; Cohen, 1988) in either direction, yet no significant difference was observed. Similarly, the present study had sufficient power to detect any bias greater than $57 \%$ on the forced choice tests. Moreover, the failure was consistent across three separate measures. Finally, evidence of a role for a common error term was consistently observed across these measures in the case of acquisition.

It is possible that the prediction error manipulation was rendered ineffective in the extinction component by some process unique to extinction learning. One candidate process, reinstatement, was addressed in Experiment 2.

\section{Experiment 2}

The common error term in the extinction components of Experiment 1 was manipulated by extinguishing a target cue (e.g., B) in compound with an already extinguished cue (A), and comparing test performance to $\mathrm{B}$ with that to a second target cue (D) extinguished in compound with a nonextinguished cue (C). It was assumed that the common error term was greater in the compound that consisted of two nonextinguished cues (CD) than in the compound that included an extinguished cue (AB). However, animal studies have shown that the learning produced by extinction is controlled by "context." For example, fear responding is depressed when a learned danger signal is tested in the context in which it had been extinguished, but is reinstated when tested either in the presence of or shortly after exposure to a dangerous context (Morris, Furlong, \& Westbrook, 2004). Hence, the presence of the causal cue $(B)$ in compound with the extinguished cue (A) may have acted to reinstate the 
association between A and the outcome. That reinstatement of the A-outcome association may have made the size of the error of $\mathrm{AB}$ similar to that of $\mathrm{CD}$ across the extinction trials in Phase 3, leading to similar amounts of change to B and D. The training data from Experiment 1 (Fig. 1) support this prediction: Participants treated compound $\mathrm{AB}$ as similar to compound $\mathrm{CD}$ across their respective extinction trials.

The primary aim of the present experiment was to examine this explanation by manipulating the common error term using cues that had been trained and extinguished as well as cues that had always been safe. The design is shown in Table 2. In Phase 1, Mr. X suffered from an allergic reaction after ingesting each of six different foods (A-D, F, L) but not after ingesting seven other foods $(\mathrm{E}, \mathrm{G}-\mathrm{K}, \mathrm{M})$. In Phase 2 , one of the allergenic cues was extinguished (A-). In Phase 3, three compounds were extinguished: AB-, CD- and EF-. Thus, B was extinguished in compound with an already extinguished A, D was extinguished in compound with the nonextinguished $\mathrm{C}$, and $\mathrm{F}$ was extinguished in compound with the safe E.

Finally, participants were given forced choices between three pairs of compounds. Each of these test compounds consisted of one of the target cues B, D, or F, and a reliably safe cue $(\mathrm{G}, \mathrm{H}$, or $\mathrm{I}$, respectively). If extinction is regulated by a common error term, then the amount of change should be ordered $\mathrm{D}>\mathrm{B}>\mathrm{F}$ since the predictive values of their associates across the compound extinction trials were ordered C (it was allergenic), A (it was extinguished), and E (it was safe). Hence, forced choice should be ordered FI $>$ BG $>$ $\mathrm{DH}$. Alternatively, if the reinstatement hypothesis is correct,

Table 2 Design of Experiment 2

\begin{tabular}{llll}
\hline Mr. X & & & \\
\hline Phase 1 & Phase 2 & Phase 3 & Test 1 Test 2 \\
\hline A + & A- & AB- & BG vs. DH \\
B+ & & & DH vs. FI \\
C+ & & CD- & BG vs. FI \\
D+ & & & \\
E- & & EF- & \\
F+ & & & \\
G- & G- & & \\
H- & H- & & \\
I- & I- & & \\
J- & J+ & JK + & \\
K- & K+ & LM+ & \\
L+ & L+ & & \\
M- & M+ & & \\
\hline
\end{tabular}

Letters A-M denote cues, and "+" and "." indicate reinforced and nonreinforced trials, respectively. Participants witnessed all trial types within each phase presented in a randomly intermixed fashion then the amount of change to D and B should be similar since the presence of $B$ would have restored the predictive value of its extinguished A associate. Again, however, the amount of change to $\mathrm{D}$ and $\mathrm{B}$ should be greater than that to F. Hence, forced choice should be ordered $\mathrm{FI}>\mathrm{BG}=\mathrm{DH}$.

Finally, in Experiment 1, participants were asked to choose which of two compounds was more dangerous for Mr. X when each of these compounds contained an extinguished and an allergenic element. It is therefore possible that any small changes in extinction may have been overshadowed by the presence of the allergenic cues at test. Alternatively, the presence of a causal cue at test may have acted to reinstate the earlier causal status of cues extinguished in Phase 3. In the present experiment, we excluded these possibilities by pairing each of the target cues $(B, D$, and $F)$ with a safe cue $(\mathrm{G}, \mathrm{H}$, and $\mathrm{I})$ at test.

\section{Method}

Participants Thirty postgraduate psychology students (mean age 25 years) volunteered to participate in the experiment.

Procedure The procedure for Experiment 2 was identical to that in Experiment 1, with one exception. In the test phase of Experiment 2, there were three forced choice test trials. The order of these trials was randomized for each participant. They were otherwise identical to those in Experiment 1.

\section{Results}

Training data The prediction data for acquisition of the cue-allergy and cue-no allergy associations in Phase 1 and for extinction of the cue-allergy associations in Phases 2 and 3 are shown in Fig. 3. Participants quickly learned which cues (including A, B, C, D, and F) predicted the allergy and which (including E) were safe in Phase 1. They also changed their predictions about $\mathrm{A}$ in Phase 2, coming to correctly predict that it no longer signaled the allergy. Predictions about the $\mathrm{AB}, \mathrm{CD}$, and $\mathrm{EF}$ compounds were similar in Phase 3. Initially, participants predicted the allergy on presentation of each of these compounds and changed their predictions to each in a similar manner across the compound extinction trials, coming to correctly predict that the compounds were not followed by the allergy.

Forced choice data There were no statistically significant differences in the proportion of participants selecting one or another of the compounds in any of the three pairs, $p>.05$. Participants were just as likely to select BG (18 out of 30$)$ as $\mathrm{DH}$, in spite of the fact that $\mathrm{B}$ had been extinguished in compound with the already extinguished A, whereas D had 
Fig. 3 Outcome predictions for cues A-F during Phases $1-3$ in Experiment 2

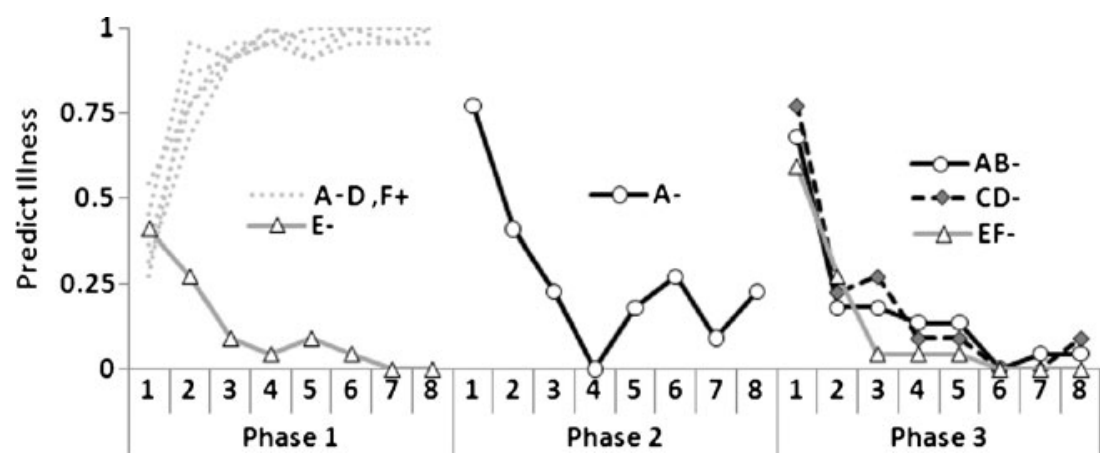

been extinguished in compound with another allergen, $\mathrm{C}$. The failure to detect a difference in the selection of the compounds on test could have been due to the restoration by $B$ of the allergenic properties of its partner cue (A) across the compound extinction trials. This restoration would have made the error term for $\mathrm{AB}$ similar to that of $\mathrm{CD}$, thereby producing similar change to $\mathrm{B}$ and $\mathrm{D}$. However, this change should have been greater than that accruing to $\mathrm{F}$ extinguished in compound with the safe E. Hence, FI should have been selected as more causal than either BG or DH. But this did not occur. Participants were indifferent in each of these choices.

Causal ratings Mean causal ratings for all cues are shown in Fig. 4. Five orthogonal contrasts were used to analyze the causal ratings data. The cues paired with the outcome (J-M) were rated as more causal than the safe or extinguished cues $(\mathrm{A}-\mathrm{I}), F(1,28)=73.04, p<.001, \eta_{\mathrm{P}}{ }^{2}=0.722$, and the safe cues (E, G, H, and I) were rated as less causal than the cues first paired with the outcome and then extinguished $(\mathrm{A}, \mathrm{B}$, $\mathrm{C}, \mathrm{D}$, and F), $F(1,28)=121.41, p<.001, \eta_{\mathrm{P}}{ }^{2}=0.813$. Consistent with Experiment 1, the amount of extinction regulated judgments of effectiveness. The cue extinguished in Phases 2 and 3 (A) was rated as significantly less causal than the average of the cues $(B, C, D, F)$ that were extinguished only in Phase $3, F(1,28)=9.52, p=.005, \eta_{\mathrm{P}}{ }^{2}=$ 0.253 . Critically, the cue (D) extinguished in compound with another causal cue (C) was not rated as significantly

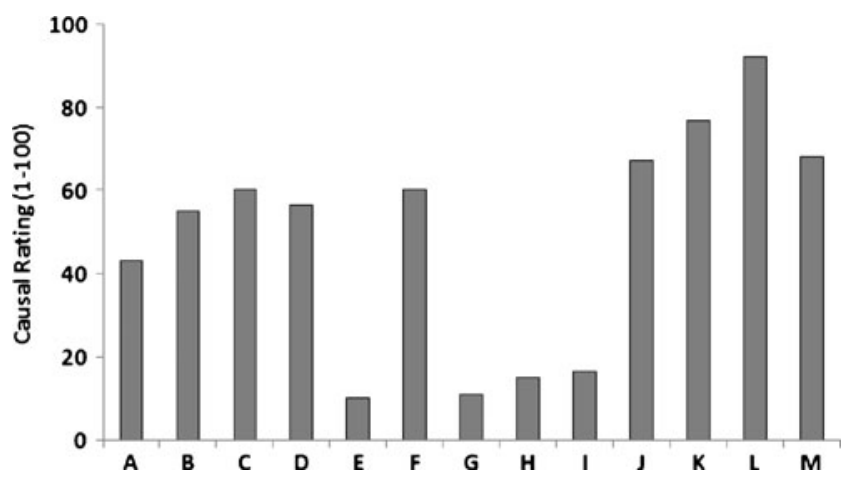

Fig. 4 Mean causal ratings in Experiment 2 less causal (indicating more extinction learning) than the average of cues (B and F), one of which (B) having been extinguished in compound with an already extinguished cue (A) and the other $(\mathrm{F})$ having been extinguished in compound with a safe cue (E), $F<1$. Moreover, the cue (B) extinguished in compound with an already extinguished cue (A) was not rated significantly differently to the cue $(F)$ extinguished in compound with a safe cue (E), $F<1$. This finding, in particular, is inconsistent with the reinstatement hypothesis.

\section{Discussion}

In the present experiment, we again manipulated prediction error by extinguishing the allergenic $\mathrm{B}$ in compound with the already extinguished A, versus extinguishing the allergenic $\mathrm{D}$ in compound with the allergenic $\mathrm{C}$. The previous failure to detect a difference between $B$ (extinguished in compound with the already extinguished A) and D (extinguished in compound with the allergenic C), could have been due to the effects of B on A. Specifically, the threat posed by $B$ could have reinstated the allergenic properties of the extinguished $\mathrm{A}$, thereby rendering $\mathrm{AB}$ and $\mathrm{CD}$ effectively equivalent with respect to error across their extinction. In the present experiment, we assessed this possibility by including a condition in which the allergenic $\mathrm{F}$ was extinguished in compound with the safe E. However, extinction of the target cue $(\mathrm{B}, \mathrm{D}$, or $\mathrm{F})$ was again unaffected by the predictive value of its associate in the compound (A, C, and E, respectively), in spite of the fact that the manipulations used to vary predictive value were successful. The causal ratings confirmed that cue (A) extinguished in Phases 2 and 3 was rated as less effective than those only extinguished in Phase 3 (e.g., C), and, in turn, those extinguished in Phase 3 were rated as more causal than the safe cues (e.g., E). Moreover, the previous failure to detect any differences on the compound tests could have been due to reinstatement of the effectiveness of the extinguished target by its dangerous partner at test. However, in this experiment, we again failed to detect differences when compounds that contained the target cue $(B, D$, and $F)$ and a safe cue $(G, H$, 
and I, respectively) were pitted against each other at test. Finally, the test data are consistent with the online outcome predictions, which were similar across extinction of the $\mathrm{AB}$, $\mathrm{CD}$, and $\mathrm{EF}$ compounds in spite of the fact that $\mathrm{A}$ had just been extinguished (its ratings were low) and $\mathrm{F}$ was safe (its ratings were also low), whereas $\mathrm{C}$ and $\mathrm{D}$ were each dangerous (their ratings were high). Thus, participants did not appear to use the information about the partner cue to regulate either their predictions across extinction of the compounds or the amount learned about their target cue.

\section{Experiment 3}

A curious feature of the data in Experiment 1 and 2 is that the causal ratings given to the extinguished cues at test were moderate (48-59 out of 100), whereas terminal responding for these cues during training was essentially at floor. One possible explanation for this mismatch is that participants tend to aggregate across their entire training experience when giving final ratings, but do not tend to do so for online measures (Collins \& Shanks, 2002). An alternative explanation, however, is that the moderate ratings at test are the more accurate measure of learning. Recall that the online predictions during training were binary, whereas the final test ratings were continuous. This raises the possibility that the very low level of responding during training reflect moderate associative strength values that were nevertheless below some threshold level required for an "allergy" prediction. On this view, these moderate levels of associative learning were then accurately reported on the continuous outcome prediction scale at test. If indeed extinction training of A had merely resulted in some (but by no means complete) learning of its new relation with the allergy, then its ability to block learning about $\mathrm{B}$ across the $\mathrm{AB}$ compound extinction trials would be reduced, and hence ratings of $B$ would be similar to those of $\mathrm{D}$ extinguished in compound with the allergenic C. However, ratings of B and D should have been lower than those of $\mathrm{F}$, which had been extinguished in compound with the safe E. However, this did not occur. Nevertheless, it seemed important to assess this explanation of the failure to detect differences between $\mathrm{B}$ and $\mathrm{D}$ in the previous experiments. Hence, in Experiment 3, we used a continuous outcome measure at both training and test. Doing this allowed for a more direct comparison of outcome predictions at training and test and, importantly, provided a better measure as to whether extinction learning reached asymptote in training.

Two additional changes to the test procedure were implemented to increase its sensitivity to the experimental manipulations. First, in Experiment 3, we replaced the compound test procedure with an elemental forced choice test procedure. This procedure more directly assesses the comparison of interest (between cues B and D) than the compound test procedure used in Experiment 1 and 2. Second, the test procedure included explicit instructions for participants to make their ratings according to the current predictive value of the cue, rather than to aggregate across their prior training experience.

\section{Method}

Participants Sixty-eight undergraduate psychology students (mean age 20 years) completed the experiment as part of their coursework.

Procedure The procedure for Experiment 3 was very similar to that in Experiment 1, so only the differences will be detailed. First, participants were given only one patient, Mrs. X, and the cover story was changed slightly. Participants were initially told the following:

"It is your job to assume the role of a doctor who specialises in food allergies. You have a new patient (Mrs X) who is currently undergoing chemotherapy. She has come to you because the chemotherapy is affecting her immune system, resulting in a strange collection of food allergies. In fact, her allergies appear unstable, perhaps as a result of her constantly changing drug regimen. This means that she sometimes acquires food allergies spontaneously, and sometimes food allergies will suddenly disappear. She has come to you to try and learn which foods she should avoid. To help diagnose her specific allergies, you've provided Mrs X with a blood analyser that measures her antibody levels (a measure of allergic reaction severity). You have asked Mrs X to eat a simple diet, and record (i) the foods she eats and (ii) the antibody levels that these foods produce. Mrs X has meticulously documented all foods and antibody measurements over a four month period.

You will review this information. To test yourself along the way, after each meal, you'll be asked to predict whether Mrs X will have a reaction, and if so, how bad that reaction will be. Also, you'll be asked to rate how confident you are of that prediction (on a scale of 1 to 5)."

Online outcome predictions were provided on a continuous allergen severity scale (similar to that used in Griffiths, Johnson \& Mitchell, 2011). Participants were given access to this during the instructions so as to familiarize themselves with this response format prior to the experiment. The scale ranged from $0-6$, in increments of 0.1. The range from $0-2$ was labeled none and was colored green, $2-4$ was labeled 
minor and was colored orange, and 4-6 was labeled serious and was colored red. Every time the position of the indictor on the rating scale was adjusted, participants were shown the categorical (e.g., minor) and numeric (e.g., 0.8) prediction that this position indicated. The color of the indicator also changed to reflect the categorical prediction (in the aforementioned example, it became green).

After participants had familiarized themselves with the response measure, they began training. The design of the training procedure closely resembled that of Experiment 1, and is summarized in Table 3. On each trial, one or two foods were shown (elemental or compound trials), and participants were required to predict the severity of Mrs. X's allergenic reaction. They did so by manipulating the allergen rating scale. They were also required to rate their confidence in their prediction on a second 1-5 confidence scale. The extremes were labeled not at all confident and very confident. Feedback was then provided. Participants were shown a second allergen scale with the correct answer shown. For a "no allergy" trial, the indicator on the feedback scale was randomly positioned between 0.4 and 1.3 (within the none range). On an "allergy" trial, the indicator on the feedback scale was positioned between 4.6 and 5.5 (in the serious range). The second scale was located next to the scale on which they made their prediction, to allow for direct visual comparison. After $2 \mathrm{~s}$, the second scale disappeared, and a new trial began. There were eight repetitions of each trial type in every phase.

The test phase was preceded by the following instructions:

"You've now studied all of Mrs X's records. You should have a fairly good idea of which foods make her feel ill, and which do not. This is important because Mrs X is about to undergo a particularly difficult

Table 3 Design of Experiment 3

\begin{tabular}{llll}
\hline Phase 1 (8) & Phase 2 (8) & Phase 3 (8) & Test 1: \\
\hline A+ & A- & AB- & A vs. C \\
B + & & CD- & B vs. D \\
C+ & & & \\
D + & & & \\
E- & E+ & & \\
F- & F- & GH+ \\
G- & G- & & \\
H- & H- & & \\
I- & I+ & J+ & \\
J+ & J+ & & \\
& K+ &
\end{tabular}

Letters A-K denote cues, and "+" and "-”" indicate reinforced ("serious allergy") and nonreinforced ("no allergy") trials, respectively. Participants witnessed all trial types within each phase eight times. They were presented in a randomly intermixed fashion treatment, during which time it is vitally important that she doesn't experience any allergies at all. That is, it is important to determine which foods are likely to be dangerous to her right now.

First, the hospital has provided Mrs X with a choice of two meals. You must choose which of these is least likely to make her feel ill. Once that decision is made, you will be asked to advise her regarding how dangerous the other foods are to her now."

The test phase then began with a forced choice between cues B and D. Participants were asked to choose which meal would be least likely to make Mrs X ill. They were then forced to choose between foods A and C. After these choices were made, individual cue ratings were collected for each cue. These ratings were made on a scale from $0-6$, with extremes labeled very [un]likely to have an allergic reaction next time Mrs $X$ eats it. The cues were rated in a random order.

\section{Results}

Training data Participants' mean outcome predictions and confidence ratings are plotted in Fig. 5. The columns refer to confidence ratings, whereas the lines refer to outcome predictions. Participants gradually increased their outcome predictions for cues A-D in Phase 1, such that they were reliably, correctly providing predictions of a "serious" outcome midway through Phase 1. Confidence increased accordingly across Phase 1. Then, in Phase 2, participants rapidly extinguished to cue $\mathrm{A}$, such that their predictions reached an asymptotic value within the correct no allergy range by the fourth A- trial. These asymptotic predictions were offered with high confidence. Finally, in Phase 3, participants rapidly extinguished to compounds $\mathrm{AB}$ and $\mathrm{CD}$, so that by the fourth trial, participants reliably predicted a value within the correct no allergy range for these compounds. The higher predictions for compound $\mathrm{AB}$, than for $\mathrm{CD}$, on Trial 1 of Phase 2 did not reach significance, $t(67)=$ $1.88, p=.06$. Confidence began high and gradually increased across Phase 3.

Forced choice data If a common error term regulated extinction learning, participants should have learned more about D than B; consequently, D should have been chosen as safer than B. Two-tailed binomial tests were used to analyze this choice. Thirty-one of 68 participants showed this preference. This was not significantly different from chance, $z=0.73, p>.05$. However, when forced to choose between cues $A$ and $C$, significantly more participants chose the repeatedly extinguished cue A (44) as safer than the singly extinguished cue C (24), $z=2.43, p=.02$. 


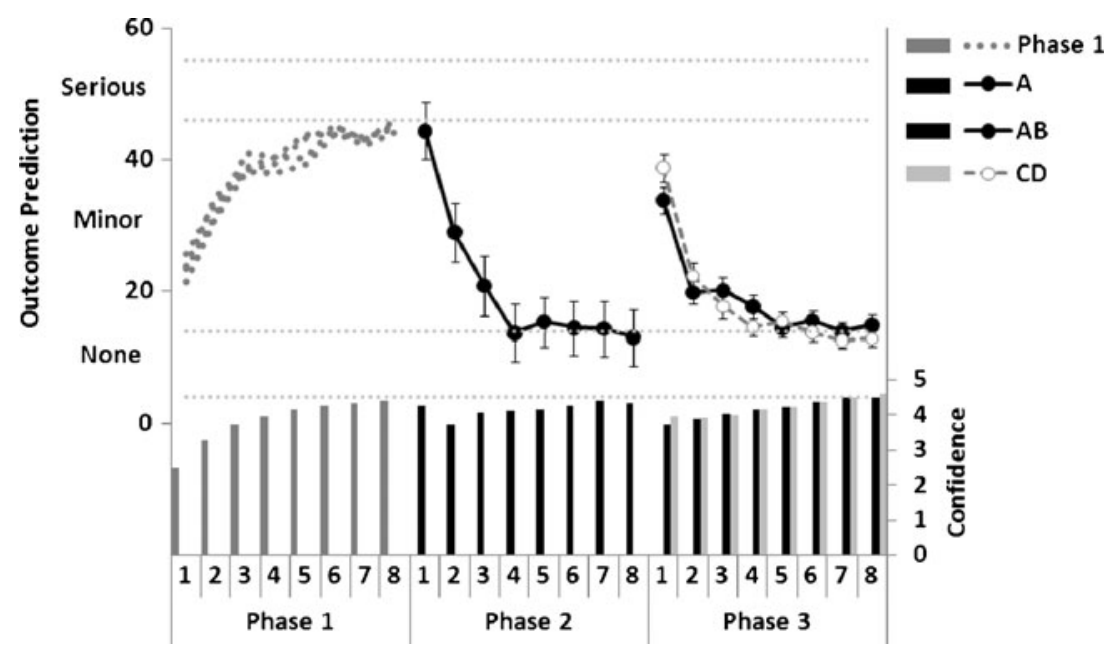

Fig. 5 Outcome predictions (lines) and confidence ratings (columns) for cues A-D in Experiment 3. The lines refer to mean outcome predictions for each cue type and are plotted with respect to the lefthand axis. The light, horizontal broken lines indicate the range of outcome values that occurred on trials involving a "serious allergy" (the upper values) or a "no allergy" outcome (the lower values). The

Causal ratings Participants' ratings of the individual cue are summarized in Fig. 6. Three orthogonal contrasts were tested in a multivariate ANOVA procedure. First, the repeatedly extinguished cue A was given significantly lower ratings than the average of the singly extinguished cues $\mathrm{B}, \mathrm{C}$, and $\mathrm{D}, F(1,67)=6.72, p=.012, \eta_{\mathrm{P}}{ }^{2}=0.091$. Critically, no significant difference was seen between $\mathrm{B}$, which was extinguished in compound with the previously extinguished A, than the average of cues $\mathrm{C}$ and $\mathrm{D}$, each of which was allergenic when extinguished in compound, $F<1$. A final contrast confirmed that there were no systematic differences in the ratings given to cues $\mathrm{C}$ and $\mathrm{D}, F<1$.

\section{Discussion}

In Experiment 3, we again failed to detect any evidence for the use of a common error term in extinction learning. Participants did not differ in the rate at which they changed

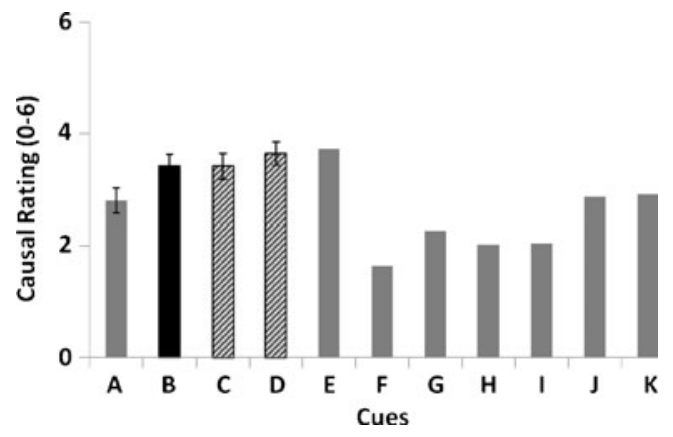

Fig. 6 Mean causal ratings in Experiment 3. The critical comparisons are represented by dark and striped columns
Phase 1 data for the various cue types are not differentiated, since they were all treated identically during this phase. The columns depict the mean confidence ratings for each cue type. These are plotted against the right-hand axis. Phase 1 confidence data were aggregated across all cue types, since the cues were treated identically in this phase. Error bars indicate the standard errors of the means

their predictions or in the confidence of these predictions across extinction of $\mathrm{AB}$, a compound composed of the allergenic $\mathrm{B}$ and the already extinguished $\mathrm{A}$, or of $\mathrm{CD}$, a compound composed of the allergenic $\mathrm{C}$ and the allergenic D. Nor did they rate B as more allergenic than $\mathrm{C}$ or $\mathrm{D}$, which they should have done if $\mathrm{A}$ had blocked extinction learning about B. This failure to detect an effect of A on B occurred in spite of the fact that participants selected $A$ as safer for consumption than $\mathrm{C}$ when forced to choose between them, and rated $\mathrm{A}$ as less effective in producing an allergy than $\mathrm{B}, \mathrm{C}$, and $\mathrm{D}$ when required to rate all of the cues.

\section{General Discussion}

In the present experiments, we used a variant of the blocking effect to assess the role of error correction mechanisms in the acquisition and extinction of causal judgments in people. In all three experiments, a single cue (A) was extinguished in isolation. Then, A received additional extinction in compound with the allergenic $\mathrm{B}$, while two other allergenic cues$\mathrm{C}$ and D-were also extinguished in compound. The prediction error on the compound extinction trials should be lower on $\mathrm{AB}$ - trials, than on $\mathrm{CD}$ - trials, because of the prior extinction of $\mathrm{A}$. If a common error term regulates extinction learning, this should lead to reduced extinction learning for cue B, relative to cue D. No such effect was seen across the three experiments. The failure to observe this effect is unlikely to be due to experimental insensitivity. In Experiment 1, we observed a comparable "blocking" effect in acquisition learning under the same experimental parameters (on three separate measures), which shows that the procedure is 
sensitive to differences in learning because of the common error term manipulation. Furthermore, in all three experiments, we confirmed that participants rated the cue, Aextinguished in isolation and then in compound-as less causally effective than cues (e.g., C) just extinguished in compound with another allergenic cue. This result shows that the procedure was sensitive to differences in extinction learning. However, as was just noted, none of the experiments provided evidence that extinction learning was modulated by a common prediction error term.

Two explanations as to why cue competition was absent in extinction were experimentally addressed. First, the previously extinguished cue A may have been reinstated in the compound extinction phase by the presence of the allergenic cue B. Such an effect would serve to equalize the error terms between that compound, $\mathrm{AB}$, and $\mathrm{CD}$ across the compound extinction trials; consequently, no differences between cues $\mathrm{B}$ and $\mathrm{D}$ would be anticipated. This explanation was examined in Experiment 2 by extinguishing cue $\mathrm{F}$ in compound with the safe cue E. Because cue $\mathrm{E}$ was never paired with the allergy outcome, it could not undergo reinstatement when paired with the allergenic cue $F$. This should have freed cue $\mathrm{E}$ from reinstatement effects and consequently impaired extinction learning for cue $\mathrm{F}$ (relative to cue $\mathrm{D}$, or B). However, consistent with the results of other experiments, no differences in extinction learning were seen between cues $\mathrm{B}, \mathrm{D}$, or F, suggesting that a reinstatement effect did not mask the influence of the common error term on extinction.

Alternatively, it is possible that extinction learning of A was incomplete; hence, error across the subsequent $\mathrm{AB}$ extinction trials would have been large, again serving to reduce the differences in the size of the error between $A B$ and $\mathrm{CD}$, thereby promoting just as much extinction to $\mathrm{B}$ as to D. In Experiment 3, we addressed this hypothesis by using a continuous measure during training, and we found that participants' online outcome predictions stabilized on a low outcome prediction value midway through extinction training (and these predictions were offered with high confidence). This pattern of behavior is indicative of asymptotic extinction learning about A. Even so, no cue competition effects were observed in extinction. Finally, it is possible that extinction learning about A was at asymptote in advance of its additional extinction in compound, but failed to block extinction learning of $\mathrm{B}$ because it did not undergo an additional increase in associative change across the compound extinction trials. Models such as Rescorla-Wagner predict that additional extinction of $\mathrm{A}$ in compound with the nonextinguished B will simultaneously convert A into a net inhibitor or preventative cause of the allergy and reduce extinction to B. The present experiments were not designed to assess whether A had in fact developed such inhibition. However, if A failed to develop such inhibition, remaining instead at a relatively low associative value, then its ability to block extinction of its
B associate would have been reduced. As far as we are aware, this prediction regarding the development of inhibition to a cue (A) first extinguished in isolation and then in compound with another cue (B) remains to be examined.

As was noted in the introduction, models such as Rescorla-Wagner (Rescorla \& Wagner, 1972) and PearceHall (Pearce and Hall, 1980) assume that a common error term regulates associative change in both acquisition and extinction. According to these models, therefore, the presence of an already extinguished cue should have blocked learning about its associate across compound extinction trials just as the presence of an already trained cue blocked learning about its associate across the compound conditioning trials. Other models of associative formation that do not incorporate a common error term cannot easily explain the present data either. For example, Mackintosh's (1975) model does not include a common error term, yet can explain many of the effects for which a common error term was proposed (e.g., blocking). The Mackintosh model explains blocking $(\mathrm{A}+$ then $\mathrm{AB}+)$ by proposing that the pretrained cue A blocks its associate (B) across reinforced compound trials $(\mathrm{AB}+)$ because attention is maintained to cue $\mathrm{A}$, but not to cue $\mathrm{B}$. In this model, attention is maintained to $\mathrm{A}$ because the prediction error associated with $A\left(\lambda-V_{A}\right)$ is less than that associated with $B\left(\lambda-V_{B}\right)$. Importantly, this model predicts that in the present manipulation (A- then AB-), the already extinguished cue A should block its associate (B) across nonreinforced compound trials because cue $A$ is a better predictor of the new outcome $\left(V_{A}\right.$ is close to the new $\lambda$ of zero) than is cue $B\left(V_{B}\right.$ is high). Hence, attention to cue $B$ should be reduced, and its association with the outcome preserved, relative to the erasure of the association between cue $\mathrm{D}$ and the allergy outcome across the CD- extinction trials.

Like other classic models (e.g., Bush \& Mosteller, 1951; Estes, 1950: Rescorla \& Wagner, 1972), Mackintosh's (1975) model explains extinction as the weakening and eventual erasure of the CS-US association. This explanation is now known to be incomplete or wrong (see Bouton, Westbrook, Corcoran, \& Maren, 2006, for review). However, the "extended Mackintosh model" developed by Le Pelley (2004) incorporates inhibitory learning and thus offers a better account of extinction. This extended model uses a common error term to determine the type (excitatory or inhibitory) as well the amount of learning on a given trial, while still preserving the role of individual error terms in regulating associative change. However, the use of a common error term to explain conditioned inhibition and, by implication, the learning produced by extinction, entails the same prediction as the original Mackintosh model. Specifically, both predict that a cue (B) extinguished in compound with an already extinguished cue (A) would undergo less inhibitory learning than a cue (D) extinguished in compound with another trained cue (C). 
Thus, the absence of cue-competition in human extinction learning is inconsistent with most models of associative learning, and also with the existing animal data described earlier. This pattern of findings, however, does have a precedent. Lovibond, Davis, and O'Flaherty (2000) used electrodermal conditioning to examine human extinction learning. In their second experiment, one cue $(C)$ was extinguished in compound with a conditioned inhibitor, while a second cue (D) was extinguished in compound with a previously reinforced cue. If extinction learning was modulated by the common error term, then conditioning of cue $\mathrm{C}$ ought to have been impaired, because of the presence of the conditioned inhibitor. This "protection from extinction" effect was observed in skin conductance and threat expectancy measures, since cue $\mathrm{C}$ was still perceived as dangerous at test. More importantly, common error term models also predict a "super extinction" effect for cue D. That is, they predict that the presence of a second dangerous cue ought to facilitate extinction learning for cue D. In fact, the opposite effect was found: Pairing cue $\mathrm{D}$ with another dangerous cue during extinction impaired extinction for cue D. Integrating Lovibond et al.'s (2000) results with those reported presently, it appears that in human contingency learning, the presence of any additional cue during extinction training affords some protection to its partner cue, irrespective of the summed error term.

How can this asymmetry between human acquisition and extinction learning, which differs markedly from that seen in animal studies, be integrated with standard associative accounts of learning? One possibility is that humans are more sensitive to context (temporal, spatial, and internal) than animals, and that they are particularly sensitive when undergoing extinction learning. In order to explain the present data within this framework, it is necessary to posit that extinction is so acutely context sensitive that any variation between trials during extinction training (and test) will produce a severe decrement in generalization of extinction learning.

The present training data offer some support for this interpretation. Notice that in the training data from all three experiments, prior extinction training with cue A (A- trials) did not significantly reduce the initial outcome predictions for compound $\mathrm{AB}$ relative to compound $\mathrm{CD}$. Instead, compounds $\mathrm{AB}$ and $\mathrm{CD}$ were treated identically throughout the compound extinction phase. It is almost as if participants disregarded their prior experience with cue A when assessing compound $\mathrm{AB}$. If so, then this could explain the equivalence of cues B and D observed at test because, on this view, the extinction treatment of cues B and D (in compounds $\mathrm{AB}$ - and $\mathrm{CD}$-, respectively) is identical. This failure to generalize between the elemental A- extinction trials and the compound AB- extinction trials could be considered an extreme form of context-specific learning. Specifically, the presence of cue $\mathrm{B}$ on $\mathrm{AB}$ - trials could act as a change of context of sufficient magnitude to render prior learning for cue A alone irrelevant. This hypothesis may also explain the modest test ratings given to the singly extinguished cues (e.g. B, C, and D), relative to the low final ratings given to the repeatedly extinguished cue A. The singly extinguished cues were only extinguished in compound, which may not transfer effectively to the elemental test procedure. In contrast, the repeatedly extinguished cue A was also extinguished in isolation and thus was given lower causal ratings in the elemental test procedure.

Alternatively, a recent state-based model may also explain the present data (Redish, Jenson, Johnson, \& KurthNelson, 2007). It holds that participants learn the value of each cue via an error-based temporal difference algorithm (Sutton \& Barto, 1981), which is conceptually similar to the Rescorla-Wagner rule. It is this component that accounts for the well-documented effects of prediction error on acquisition and is confirmed here. However, the model includes a second component that organizes and stores different "states" and, critically, creates new states. It is this "state-splitting" that explains the asymmetries between acquisition and extinction seen here. Specifically, the proposal is that chronic negative prediction error (as in extinction), but not positive prediction error (as in acquisition), tends to create a new state. Thus, this model, like that proposed by Capaldi (1966), views the shift from acquisition to extinction in terms of discrimination, supposing that the negative prediction error results in participants viewing these new contingencies in terms of a new state.

Importantly, the production of a new state depends upon the dissimilarity of the present context with that of previously experienced ones, rather than upon prediction error per se. This means that if the dissimilarity between extinction training and prior training is greater than some threshold value, then a new state is formed and learning proceeds about that new state. The prediction error that drives the temporal difference learning mechanism, which calculates the value of a cue in a given state (and thus produces extinction learning), is then calculated with respect to the expected values in this new state, rather than to the value of the cue in the prior states. Indeed, if the expected outcome value of new states is initialized at zero, no new value learning is required in extinction. However, the important implication of this model is that in the present experiments, we did not actually manipulate the error term used to learn about the associative values in the new state. Rather, this error term simply determines the likelihood that a new state was formed. Instead, a different error term (the difference between the current outcome and that predicted by experience with the new state) is used to regulate extinction learning. If the initial extinction trials for all cues (e.g. AB-, CD-) were sufficiently dissimilar to previous trials (e.g. A-, $\mathrm{C}+$ ) so as to produce new states, then the amount of prediction error (as manipulated in the present experiments) would not regulate subsequent extinction learning and, consequently, this 
learning would be equivalent, which were the results observed. Thus, Redish et al. (2007) provided an account of the present data by incorporating both a prediction-error learning mechanism (temporal difference reinforcement learning) and a state-based inference mechanism (formalized using radial basis functions).

Finally, the present data could be understood within a configural theoretical framework (e.g., Pearce, 1994). The logic here is very similar to the contextual-sensitivity account discussed previously, but the terminology is different. Under a configural account, each trial type (A-, AB-) is treated as different cue. Learning about a particular cue-configures selectively generalizes to other, similar cue-configures. In order for this account to explain the data, the elemental Aextinction trials would need to be considered very dissimilar to AB- trials, such that little generalization occurred between these trial types. Ultimately, however, the configural account (like the contextual account) cannot explain why extinction learning fails to generalize from an elemental trial type (A-) to a compound trial type (AB-), whereas acquisition training readily does so $(\mathrm{A}+$ to $\mathrm{AB}+$, e.g., blocking effects).

Notice that none of these explanations explicitly rejects the role of a common error term in guiding learning. However, they substantially undermine its role. If prior learning about a cue (e.g., A) on its own does not transfer/generalize to trials that involve that same cue plus an additional cue (e.g., B), then there seems little value in summing the contribution of the individual cues on a compound-cue trial, because the contribution will be negligible. The present data, therefore, do not exclude the possibility that a common error term regulates human extinction learning, but instead suggest that its role is negligible.

In sum, the present data demonstrate a striking asymmetry in cue competition between human acquisition and extinction learning. They also highlight a discontinuity between human and animal associative learning. The data are not supportive of a role for a common-error term in the regulation of extinction. The possible explanations of the data minimize the contribution of this error term by suggesting that human extinction learning is specific to the particular situation in which it occurs. Such an explanation could take the form of a statebased or context-specific account, but the present data do not discriminate among these accounts.

Author Note This research was funded by an Australian Research Council Discovery Grant \#DP110105136.

\section{Appendix A1}

Food stimuli used as cues in the present experiments:

$$
\text { Eggs }
$$

Mushrooms
Ham
Apples
Peaches
Steak
Bread
Grapes
Noodles
Carrots
Rice
Pasta
Oranges
Onion
Tuna
Chicken
Potato
Banana
Lettuce
Spinach
Cucumber
Yogurt
Tomato
Garlic

\section{References}

Aitken, M. R. F., Larkin, M. J. W., \& Dickinson, A. (2000). Superlearning of causal judgements. Quarterly Journal of Experimental Psychology, 53B, 59-81.

Bouton, M. E. (1993). Context, time, and memory retrieval in the interference paradigms of Pavlovian learning. Psychological Bulletin, 114, 80-99.

Bouton, M. E., Westbrook, R. F., Corcoran, K., \& Maren, S. (2006). Contextual and temporal modulation of extinction: Behavioral and biological mechanisms. Biological Psychiatry, 60, 352-360.

Bush, R. R., \& Mosteller, F. (1951). A mathematical model for simple learning. Psychological Review, 58, 313-323.

Capaldi, E. J. (1966). Partial reinforcement: A hypothesis of sequential effects. Psychological Review, 73, 459-477.

Chapman, G. B., \& Robbins, S. J. (1990). Cue interactions in human contingency judgment. Memory and Cognition, 18, 537-545.

Cohen, J. (1988). Statistical power analysis for the behavioural sciences. NJ: Erlbaum.

Collins, D. J., \& Shanks, D. R. (2002). Momentary and integrative response strategies in causal judgment. Memory \& Cognition, 30, $1138-1147$.

Dickinson, A. (2001). Causal learning: An associative analysis. Quarterly Journal of Experimental Psychology, 54B, 3-25.

Dickinson, A., Shanks, D. R., \& Evenden, J. L. (1984). Judgement of act-outcome contingency: The role of selective attribution. Quarterly Journal of Experimental Psychology, 36A, 29-50.

Estes, W. K. (1950). Toward a statistical theory of learning. Psychological Review, 57, 94-107.

Griffiths, O., Johnson, A. M., \& Mitchell, C. J. (2011). Negative transfer in human associative learning. Psychological Science, 22, 1198-1204. 
Kamin, L. J. (1969). Selective association and conditioning. In N. J. Mackintosh \& W. K. Honig (Eds.), Fundamental issues in associative learning (pp. 42-64). Halifax, Canada: Dalhousie University Press.

Larkin M. J. W., Aitken M. R. F., \& Dickinson A. (1998). Retrospective revaluation of causal judgments under positive and negative contingencies. Journal of Experimental Psychology: Learning, Memory, and Cognition, 24, 1331-1352.

Le Pelley, M. E. (2004). The role of associative history in models of associative learning: A selective review and hybrid model. Quarterly Journal of Experimental Psychology, 57B, 193-243.

Le Pelley, M. E., \& McLaren, I. P. L. (2003). Learned associability and associative change in human causal learning. Quarterly Journal of Experimental Psychology, 56B, 68-79.

Le Pelley, M. E., Oakeshott, S. M., Wills, A. J., \& McLaren, I. P. L. (2005). The outcome-specificity of learned predictiveness effects: Parallels between human causal learning and animal conditioning. Journal of Experimental Psychology: Animal Behavior Processes, 31, 226-236.

Leung, H. T., \& Westbrook, R. F. (2008). Spontaneous recovery of extinguished fear responses deepens their extinction: A role for error correction mechanisms. Journal of Experimental Psychology: Animal Behavior Processes, 34, 461-474.

Lovibond, P. F., Davis, N. R., \& O'Flaherty, A. S. (2000). Protection from extinction in human fear conditioning. Behaviour Research and Therapy, 38, 967-983.

Mackintosh, N. J. (1975). A theory of attention: Variations in the associability of stimuli with reinforcement. Psychological Review, 82, 276-298.

Pearce, J. M. (1994). Similarity and discrimination: A selective review and a connectionist model. Psychological Review, 101, 587-607.

Pearce, J. M., \& Hall, G. (1980). A model for Pavlovian learning: Variations in the effectiveness of conditioned but not unconditioned stimuli. Psychological Review, 87, 532-552.

Redish, A. D., Jensen, S., Johnson, A., \& Kurth-Nelson, Z. (2007). Reconciling reinforcement learning models with behavioral extinction and renewal: Implications for addiction, relapse, and problem gambling. Psychological Review, 114, 784-805.
Rescorla, R. A. (1969). Conditioned inhibition of fear. In N. J. Mackintosh \& W. K. Honig (Eds.), Fundamental issues in associative learning (pp. 65-89). Halifax, Canada: Dalhousie University Press.

Rescorla, R. A. (1979). Conditioned inhibition and extinction. In A. Dickinson \& R. A. Boakes (Eds.), Mechanisms of learning and motivation: A memorial volume to Jerzy Konorski (pp. 83-110). Hillsdale, NJ: Erlbaum.

Rescorla, R. A. (2000). Extinction can be enhanced by a concurrent excitor. Journal of Experimental Psychology: Animal Behavior Processes, 26, 251-260.

Rescorla, R. A. (2001). Are associative change in acquisition and extinction negatively accelerated? Journal of Experimental Psychology: Animal Behavior Processes, 27, 301-315.

Rescorla, R. A. (2003). Protection from extinction. Learning and Behavior, 31, 124-132.

Rescorla, R. A., \& Wagner, A. R. (1972). A theory of Pavlovian conditioning: Variations in the effectiveness of reinforcement and nonreinforcement. In A. H. Black \& W. F. Prokasy (Eds.), Classical conditioning II: Current research and theory (pp. 64-99). New York, NY: Appleton-Century-Crofts.

Shanks, D. R. (1985). Forward and backward blocking in human contingency judgements. Quarterly Journal of Experimental Psychology, 37B, 1-21.

Sutton, R. S., \& Barto, A. G. (1981). Toward a modern theory of adaptive networks: Expectation and prediction. Psychological Review, 88, 135-171.

Van Hamme, L. J., \& Wasserman, E. A. (1994). Cue competition in causality judgements: The role of nonpresentation of compound stimulus elements. Learning and Motivation, 25, 127-151.

Wagner, A. R. (1969). Stimulus validity and stimulus selection in associative learning. In N. J. Mackintosh \& W. K. Honig (Eds.), Fundamental issues in associative learning (pp. 90-122). Halifax, Canada: Dalhousie University Press.

Wagner, A. R. (1981). SOP: A model of automatic memory processing in animal behavior. In N. E. Spear \& R. R. Miller (Eds.), Information processing in animals: Memory mechanisms (pp. 5-47). Hillsdale, NJ: Erlbaum. 